\title{
Practical example of multiple antibody screening for evaluation of malaria control strategies
}

\author{
Marie-Louise Varela ${ }^{1 \dagger}$, David Koffi2 ${ }^{2 \dagger}$, Michael White ${ }^{3}$, Makhtar Niang ${ }^{1}$, Babacar Mbengue ${ }^{5,6}$, \\ Fatoumata Diene Sarr ${ }^{4}$, André Offianan Touré ${ }^{+\dagger}$ and Ronald Perraut ${ }^{1,5,7^{*}+}$
}

\begin{abstract}
Background: Ongoing efforts to fight Plasmodium falciparum malaria has reduced malaria in many areas, but new tools are needed to monitor further progress, including indicators of decreasing exposure to parasite infection. Serosurveillance is considered promising to monitor exposure, transmission and immunity.
\end{abstract}

Methods: IgG responses to three antigen biomarkers were evaluated in a retrospective study involving: (i) surveys of 798 asymptomatic villagers from 2 Senegalese endemic settings conducted before 2002 and after the 2013 intensification of control measures, and (ii) in 105 symptomatic individuals from different settings in Côte d'Ivoire. Response to up to eight $P$. falciparum antigens, including recombinant MSP1 p9 antigen and LSA ${ }_{41}$ peptide, were analysed using multiplex technology and responses to whole P. falciparum schizont extract (SE, local strain adapted to culture) were measured by ELISA.

Results: MSP1p9 and $\mathrm{LSA}_{41} \mathrm{lgG}$ responses were shown to be relevant indicators monitoring immune status in the different study sites both from Côte d'Ivoire and Senegal. Between 2002 and 2013, individuals participating in both studies showed higher decline of sero-positivity in young (<15 years: range 12\% to 50\%) than older ( $>15$ years: no decline to 15\%) individuals from Dielmo and Ndiop. A mathematical sero-catalytic model from the complete Dielmo/ Ndiop survey was used to reconstruct declining levels of sero-positivity in more detail, demonstrating that anti-SE seroprevalence levels most accurately reflected malaria exposure in the two villages.

Conclusion: For standard screening of population immune status at sites envisaging elimination, the use of ELISAbased assays targeting selected antigens can contribute to provide important epidemiologic surveillance data to aid malaria control programmes.

Keywords: Malaria, Plasmodium falciparum, ELISA, IgG, Multiple antigens, Multiplex, MAGPIX, Ivory Coast, Symptomatic malaria, Biomarkers, Asymptomatic carriage

\footnotetext{
*Correspondence: perraut@pasteur.sn

†Marie-Louise Varela, David Koffi, André Offianan Touré and Ronald Perraut contributed equally to the work reported

${ }^{7}$ Present Address: Centre Pasteur du Cameroun, Annexe de Garoua, Garoua, Cameroun

Full list of author information is available at the end of the article
}

\begin{abstract}
Background
In recent years, the scale-up of control efforts has resulted in a major decline in malaria transmission in many regions, fueling hopes for elimination in numerous countries. Nevertheless, the malaria case load is still very high (over 200 million annually) including an estimated 236,000-635,000 deaths according to the World Health Organization (WHO) [1]. Declining transmission has been attributed to improved control policies including
\end{abstract}

(c) The Author(s) 2020. This article is licensed under a Creative Commons Attribution 4.0 International License, which permits use, sharing, adaptation, distribution and reproduction in any medium or format, as long as you give appropriate credit to the original author(s) and the source, provide a link to the Creative Commons licence, and indicate if changes were made. The images or other third party material in this article are included in the article's Creative Commons licence, unless indicated otherwise in a credit line to the material. If material is not included in the article's Creative Commons licence and your intended use is not permitted by statutory regulation or exceeds the permitted use, you will need to obtain permission directly from the copyright holder. To view a copy of this licence, visit http://creativeco mmons.org/licenses/by/4.0/. The Creative Commons Public Domain Dedication waiver (http://creativecommons.org/publicdomain/ zero/1.0/) applies to the data made available in this article, unless otherwise stated in a credit line to the data. 
rapid diagnosis and effective treatment with artemisininbased combination therapy (ACT), vector control strategies-most notably long-term insecticide-treated bed nets (LLIN), intermittent preventive treatments, and close follow-up during pregnancy [2].

Monitoring changes in malaria transmission intensity and disease prevalence through surveillance allows health authorities to evaluate control programmes and plan interventions. This welcome reduction in malaria transmission poses substantial challenges to surveillance efforts, because when transmission becomes too low, clinical surveillance and entomological inoculation rate (EIR) become insufficiently sensitive to track potential transient asymptomatic parasite carriers and infected mosquitoes with a potential risk of re-emergence of malaria from this invisible reservoir. Furthermore, in addition to the threats associated with the emergence of resistance to artemisinin in Southeast Asia and insecticides in Africa, malaria has shown rebounds in countries, such as Rwanda, Sao Tome and Principe, and Zambia, some of which were leaders in new control strategies [3]. In Senegal, longitudinal analyses of data from the villages of Dielmo and Ndiop showed that after a sharp decline in malaria cases during the 2 years following the use of ACT and LLINs, a rebound in the number of malaria cases occurred $[4,5]$. Such rebounds affect all age groups and not only young children who are usually the most susceptible, indicating that the sharp decrease of exposure resulted in a clinically observable population wide decreases in natural protective immunity $[4,6]$. These observations underline the need for increased surveillance to monitor these rapid epidemiological changes.

A key tool is sero-surveillance based on the use of Plasmodium species-specific antibodies as indicators for exposure, transmission, and immunity. Such tools have significant potential for contributing to the effectiveness of malaria control and elimination programmes [7]. Antibodies are very sensitive markers of populationlevel malaria exposure in low-transmission settings and reflect cumulative exposure over a period of time [8,9]. Although this approach was used historically as part of malaria control programmes, it has not had widespread use in part because of the lack of standardized antigens and methodology [9]. Of more than 5000 proteins expressed by Plasmodium, only a few have been examined in detail [10]. A comprehensive evaluation of candidate antigens is required to identify those antibody responses that are most sensitive for detecting changes in transmission. Studies employing protein microarrays [8] or expanded repertoires of purified antigens are beginning to address this knowledge gap, and it is likely that multiple antigens will need to be included in serologic assays $[8,9,11,12]$. Previous studies conducted in the villages of Dielmo and Ndiop [13-18] and other settings [11, 19-21] have shown associations between antibody responses against Plasmodium antigens and trends in clinical malaria in the context of stable epidemiological conditions. Some antibody responses are short-lived decreasing during the dry season while some others remain positive for years $[8,22,23]$. Recent work identified antigen markers for recent exposure contributing to precise estimates of community-wide exposure [24]. However, there is a lack of comprehensive information regarding the consequences of transmission changes on antibody responses in different age groups. In addition, few analyses were focused on symptomatic cases.

In this report, a practical example is provided as a snapshot cross-sectional picture using samples from symptomatic and asymptomatic cohorts in the Côte d'Ivoire and Senegal. A large panel of antigens were initially explored using the multiplex Luminex assay [25-27], here analysis was focused on 3 main antigen targets: (i) a preerythrocytic antigen $\mathrm{LSA}_{41}$; (ii) a merozoite antigen MSP1p19, and (iii) a whole schizont extract (SE: measured by standard ELISA) from a local strain adapted to in vitro culture [28], as a reference antigen for overall antibody response against $P$. falciparum. In the cohort from Senegal, antibody responses to six additional antigens are given with regards to the sero-catalytic models. The analysis was also focused on a subgroup of individuals from the two villages who participated to the two cross sectional studies in 2002 and 2013. It is shown that these IgG responses could differentiate settings with different epidemiological context and help characterize the decay of immunity.

\section{Methods}

\section{Study area and recruitment}

The Côte d'Ivoire study involved 163 individuals (Table 1). This included 93 patients consulting for symptomatic fever in three health centres: Korhogo, located in a savannah area with a sudanian climate, the village of Man in the Western forest and mountain area, and Abobo located in the southern part of the township of Abidjan characterized by the presence of a lagoon. In addition, 35 asymptomatic individuals from a cross sectional survey in Abobo and 35 young patients with severe malaria from the University Hospital Centre (CHU) of Abidjan were included as shown in Table 1. In these areas, the level of transmission is high with EIR around 400 infective bites/person/year [29].

The protocol of surveillance was approved by the National Committee of the Ministry of Health. Individual informed written consent was obtained from participants/parents/guardians. All clinical cases were treated and followed-up according to the standard national procedure as described $[25,26]$. 


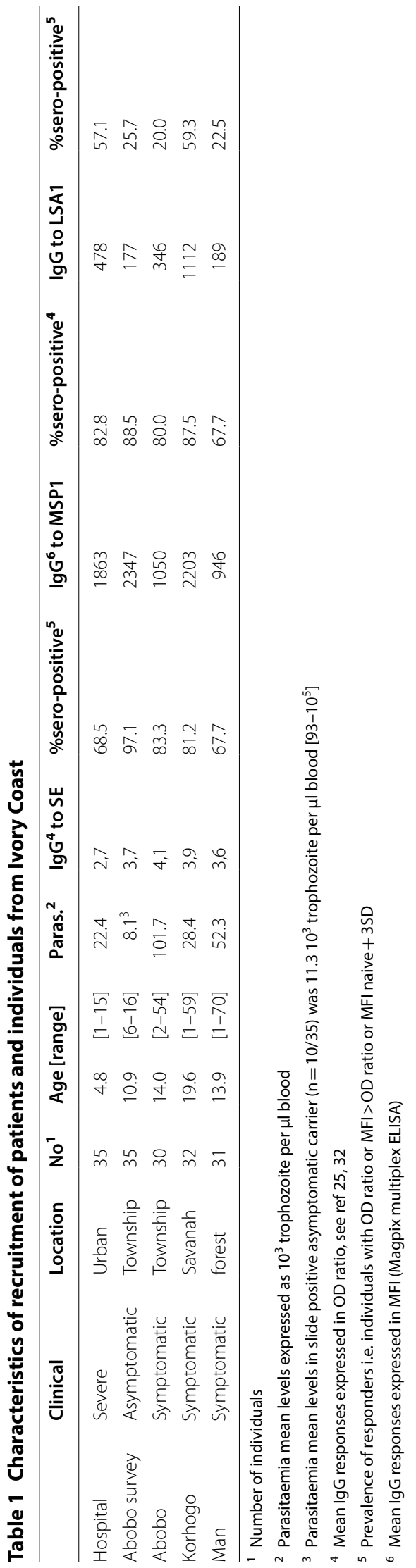


The study from Senegal was done in the endemic villages of Dielmo and Ndiop, where a long-term longitudinal survey designed to study acquisition and maintenance of natural immunity has been carried out over 20 years $[4,6,30]$. The project protocol and objectives were carefully explained to the assembled villagers, and informed written consent was obtained from all participants or their parents or guardians. Consent is individually renewed from all subjects; anyone can withdraw from the study and the follow-up procedure at any time. The protocol was approved by the Senegalese National Health Research Ethics Committee. An agreement between the Fondation Institut Pasteur de Dakar, Institut de Recherche pour le Développement (IRD) and the Ministère de la Santé et de la Prévention of Senegal defines all research activities. In July 2008, vector control was massively implemented with provision of LLINs to each household. New LLINs were provided in July 2011 [6].

Two cross-sectional samplings were done in July 2002 and July 2013 before the rainy season, i.e. before peak transmission in Dielmo (holoendemic area) and before the transmission season in Ndiop (meso-endemic). Overall 184 and 196 villagers in Dielmo, and 202 and 216 villagers in Ndiop were evaluated in 2002 and 2013, respectively with a sub-group of samples from 75 (Dielmo) and 86 (Ndiop) individuals who participated to both studies (Table 2) [27]. After withdrawal, plasma and red blood cells were separated by centrifugation and stored at $-20^{\circ} \mathrm{C}$.

\section{ELISA and Multiplex techniques for antibody responses}

The P. falciparum schizont extract (SE) of the 07/03 Dielmo strain was prepared and used in Indirect ELISA as described [31, 32]. The multiplex bead-based assay (MBA) using covalent coupled antigen (MSP1p19) or BSA-peptide $\left(\mathrm{LSA}_{41}\right)$ to carboxylated magnetic Luminex beads was done as described [33-36]. In the cohort from Senegal, antibody responses to an additional six antigens were measured using the multiplex Luminex assay: circumsporozoite protein (CSP); liver-stage antigen 3 (LSA3); glutamate-rich protein (GLURP); sporozoite and liver stage antigen (Salsa); erythrocyte associated antigen PF13 from membrane protein 1 (PfEMP1/PF13); and

Table 2 Characteristics and antibody levels and prevalence to antigens in villagers from Dielmo and Ndiop 200

\begin{tabular}{|c|c|c|c|c|c|c|}
\hline & \multicolumn{3}{|l|}{ Dielmo } & \multicolumn{3}{|l|}{ Ndiop } \\
\hline & 2002 & 2013 & $\%$ Change $^{5}$ & 2002 & 2013 & $\%$ Change $^{5}$ \\
\hline $\mathrm{No}^{1}$ & 75 & & & 86 & & \\
\hline$M / F$ & $26 / 49$ & & & $29 / 57$ & & \\
\hline $\mathrm{HbAA} / \mathrm{AS} / \mathrm{C}^{1}$ & $67 / 7 / 1$ & & & $74 / 8 / 4$ & & \\
\hline Age of Individuals & $30.3[3.8-80]$ & 41.5 [14.9-91.5] & & $26.8[3.4-68.1]$ & 37.8 [14.4-79.1] & \\
\hline $\mathrm{No}<15 \mathrm{y}^{2}$ & 20 & & & 28 & & \\
\hline $\lg G \mathrm{SE}<15 \mathrm{y}^{3}$ & $4.9[1.2-9]$ & $3.6[1-8.2]$ & $-27 \%$ & $3.6[1.0-6.7]$ & $2.5[1.0-5.4]$ & $-31 \%$ \\
\hline $\lg G \mathrm{SE}>15 y$ & $6.1[1.3-11.1]$ & $4.8[1.2-11.4]$ & $-21 \%$ & $4.7[1.0-7.8]$ & $4.1[1.0-7.1]$ & $-12 \%$ \\
\hline IgG SE all individuals & $5.8[1.2-11.1]$ & $4.5[1-11.4]$ & $-22 \%$ & $4.3[1.0-7.8]$ & $3.6[1.0-7.1]$ & $-17 \%$ \\
\hline Prev. SE $<15 y$ & $85 \%$ & $75 \%$ & $-12 \%$ & $82 \%$ & $54 \%$ & $-25 \%$ \\
\hline Prev. SE > 15y & $96 \%$ & $93 \%$ & $-4 \%$ & $84 \%$ & $84 \%$ & $0 \%$ \\
\hline $\operatorname{lgG} L S A 1<15 y^{4}$ & $1.5[0.04-9.1]$ & $0.4[0.02-1.7]$ & $-72 \%$ & $0.7[0.02-7.4]$ & $0.5[0.02-4.1]$ & $-24 \%$ \\
\hline $\lg G$ LSA1 > 15y & $1.8[0.04-12.6]$ & $1.1[0.02-6.0]$ & $-42 \%$ & $2.3[0.03-12.6]$ & $1.8[0.02-16.3]$ & $-18 \%$ \\
\hline IgG LSA1 all individuals & $1.8[0.04-12.6]$ & $0.9[0.02-6.0]$ & $-49 \%$ & $1.8[0.03-12.6]$ & $1.5[0.02-16.3]$ & $-19 \%$ \\
\hline Prev. LSA1 <15y & $80 \%$ & $75 \%$ & $-6 \%$ & $61 \%$ & $54 \%$ & $-12 \%$ \\
\hline Prev. LSA1 > 15y & $85 \%$ & $73 \%$ & $-15 \%$ & $86 \%$ & $78 \%$ & $-10 \%$ \\
\hline $\operatorname{lgG} M S P 1<15 y^{4}$ & $0.9[0.09-3.2]$ & $0.4[0.02-2.0]$ & $-58 \%$ & $1.6[0.02-12.9]$ & $1.2[0.03-12.1]$ & $-26 \%$ \\
\hline $\operatorname{lgG}$ MSP1 > 15y & $4.3[0.02-10.7]$ & $2.9[0.04-12.0]$ & $-33 \%$ & $4.1[0.2-16.2]$ & $3.3[0.05-16.6]$ & $-10 \%$ \\
\hline IgG MSP1 all individuals & $3.4[0.02-10.7]$ & $2.9[0.02-12.0]$ & $-35 \%$ & $3.3[0.02-16.2]$ & $2.6[0.03-16.6]$ & $-21 \%$ \\
\hline Prev. MSP1 < 15y & $60 \%$ & $30 \%$ & $-50 \%$ & $61 \%$ & $46 \%$ & $-24 \%$ \\
\hline Prev. MSP1 > 15y & $85 \%$ & $91 \%$ & $+3 \%$ & $88 \%$ & $79 \%$ & $-10 \%$ \\
\hline
\end{tabular}

\footnotetext{
${ }^{1}$ Number of individuals present both in the 2002 and the 2013 survey; type of haemoglobin

2 Number of individuals under 15 years old in 2002

${ }^{3} \mathrm{lgG}$ responses measured by ELISA expressed in OD ratio: mean [range]

${ }^{4} \mathrm{IgG}$ responses measured by ELISA expressed as $10^{3} \mathrm{MFI}$ : mean [range]

$5 \%$ change of prevalence/lgG levels between 2002 and 2013
} 
apical membrane antigen 1 (AMA-1) [32-36]. A pool of sera from immune adults from Dielmo and a pool of European and African non-immune sera (20 Senegalese individuals confirmed as negative IgG response to $P$. falciparum schizont extract) were included in each assay as positive and naïve controls and normalization control, respectively. For ELISA, IgG Levels were expressed as OD ratio= OD sample/mean ODnaive pool. For MBA, IgG levels were expressed as mean fluorescence intensity (MFI) or log MFI. The positivity cut-off was set as above the net signal +3 SD of naïve controls. MFI of naïve controls +3 SD ranged from 40 to 175 for the overall panel of antigens $\left(\mathrm{MSP} 1 \mathrm{p} 19=175 ; \mathrm{LSA}_{41}=63\right)$.

\section{Statistical analysis and modelling}

Categorical variables were compared using the Fisher exact test, continuous variables of antibody responses were analysed using the Man-Whitney rank test for non-normally distributed data. $\mathrm{p}$ values $<0.05$ were considered significant. Seroconversion (SCR) and sero-reversion (SRR) rates were calculated using an age-specific reversible catalytic conversion model [7]. Analyses were performed with R and Statview 5.0 ${ }^{\circledR}$ (SAS Institute).

\section{Sero-catalytic model}

Sero-catalytic models can be used to describe the rate at which individuals sero-convert following malaria exposure and subsequently sero-revert due to decaying levels of antibodies. Sero-catalytic models have typically been applied under the assumption of constant transmission over time, or a single reduction in transmission at some time in the past [7].

Here is described an extended class of sero-catalytic models accounting for multiple past changes in transmission, which can provide more flexible estimates of past transmission, as well as better accounting for uncertainty.

The sero-reversion rate is denoted $\rho . T_{\max }=80$ years was defined to be the maximum time considered before the first cross-sectional survey. The sero-conversion rate at this time is assumed to be $\lambda_{0}$. It is assumed that between $T_{\max }$ years in the past and the time of survey, the sero-conversion rate changed on $n$ occasions at times $\left(T_{1}, T_{2}, \ldots, T_{n}\right)$, so that the sero-conversion rate during the interval $\left(T_{i}, T_{i+1}\right)$ was $\lambda_{i}$. For a person of age $\alpha$ at the time of the cross-sectional survey, it is necessary to calculate their age at the times when transmission changed: $\left(\alpha_{1}, \alpha_{2}, \ldots \alpha_{m}\right)$, where $\alpha_{m}=\alpha$ (the age at the cross-sectional survey), and $m \leq n$ to account for the possibility that some of the times when transmission changed may have occurred before that person was born. It is then possible to denote $\lambda_{j}$ to be the sero-conversion rate that an individual experienced between the ages $\alpha_{j-1}$ and $\alpha_{j}$. The following recurrence equation gives the estimated proportion sero-positive at each point when the seroconversion rate changes, with $P_{m}$ being the probability that a person of age $\alpha$ is sero-positive at the time of the cross-sectional survey.

$$
\begin{aligned}
P_{1}= & \frac{\lambda_{1}}{\lambda_{1}+\rho}\left(1-e^{-\left(\lambda_{1}+\rho\right) \alpha_{1}}\right) \\
P_{j}= & \left(1-P_{j-1}\right) \frac{\lambda_{j}}{\lambda_{j}+\rho}\left(1-e^{-\left(\lambda_{j}+\rho\right)\left(\alpha_{j}-\alpha_{j-1}\right)}\right) \\
& +P_{j-1} \frac{\lambda_{j}+\rho e^{-\left(\lambda_{j}+\rho\right)\left(\lambda_{j}+\rho\right)}}{\lambda_{j}+\rho}
\end{aligned}
$$

The sero-catalytic model is parameterized by $\Theta=(\lambda 0 . . n, T 1 . . . n, \rho)$. The model is fitted in a Bayesian statistical framework with uniform prior distributions. By sampling from the estimated posterior distribution, historical sero-conversion rates in Dielmo and Ndiop can be estimated. Importantly, the inference framework provides estimation of uncertainty, allowing identification of situations when sero-conversion rates are not identifiable (e.g. it is not possible to accurately estimate seroconversion rates 40 years before a cross-sectional survey). Parameter estimates for the nine antigens are presented in Additional file 1: Table S1.

\section{Results}

\section{IgG biomarker responses and characteristics} of symptomatic malaria

In the individuals recruited in Côte d'Ivoire, only patients with severe malaria showed a significantly younger age distribution than the others $(\mathrm{p}<0.01)$ (Table 1$)$. Regarding asymptomatic individuals and severe malaria cases, parasitaemia was checked by blood smear. In the severe hospitalized cases with confirmed clinical malaria (based on WHO-defined clinical symptoms which includes RDT positive test, history of previous infection and treatment in health centre before reaching hospital), some showed negative blood smear at the time of sampling (likely due to previous treatment before/during hospitalization). In the asymptomatic cross sectional survey, $29 \%$ of individuals had no circulating parasites detectable by blood smear. Accounting only for individuals found with positive parasitaemia from asymptomatic survey and from hospitalized severe cases (detectable on blood smear), levels of parasitaemia from positive asymptomatic carriers were significantly lower than in severe cases (mean $=11300$ vs 41300 trophozoite per $\mu$ l of blood; $\mathrm{p}<0.01$ ). In patients from the three health centres, those from the township of Abobo had the highest levels of parasitaemia (Fig. 1). 

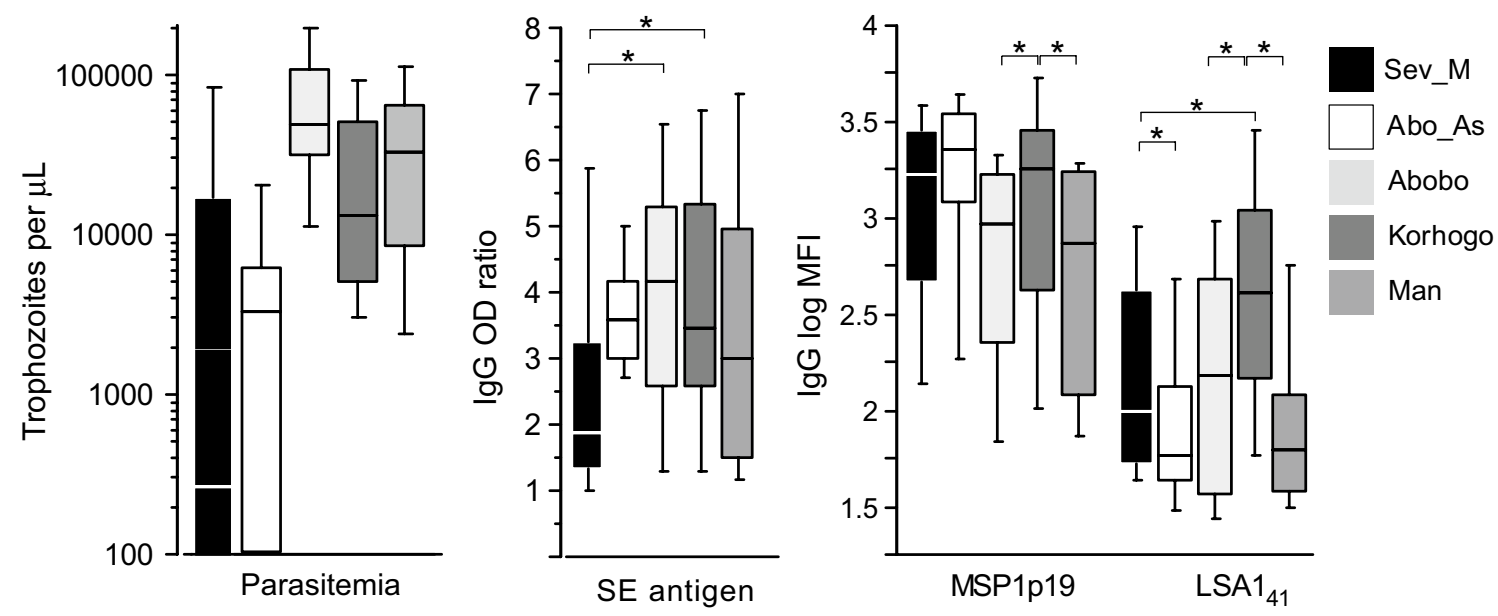

Fig. 1 Comparison of parasitaemia and distribution of antibody responses in different groups from Ivory Coast. Parasitaemia and antibody responses from individuals with urban severe malaria (Sev_M, Black), asymptomatic survey (Abo_As, white), symptomatic cases from Abobo (Abobo, light grey), from Korhogo (Korhogo, dark grey) and Man (Man, middle grey) are plotted as boxplots. Asterisks indicate significant different levels of IgG to SE, MSP1p19 and LSA1 $41(p<0.05)$

There was little difference between cohorts when comparing IgG responses to SE, except for SM cases with significantly lower levels of antibodies to SE compared to symptomatic cases in Abobo and Korhogo. This finding may be attributable to the younger age of this group.

High levels of MSP1p19 were found for Korhogo, SM and asymptomatic carriers. IgG to $\mathrm{LSA}_{41}$ was more variable among groups: Korhogo patients showed significantly higher levels than all other. The significant lowest level of anti-LSA $1_{41}$ was in asymptomatic carriers and in Man (Fig. 1).

\section{Individual decrease of antibody levels in asymptomatic villagers of Dielmo and Ndiop}

The IgG responses of the subgroup of villagers that were present and sampled in 2002 and 2013 are shown in Fig. 2. Changes in IgG levels between 2002 and 2013 for each individual are plotted as dot plot connected by arrows coloured in black when an increase in antibody response was observed. The majority, but not all individuals, showed reduced antibody responses to the three antigens.

Decreases were checked across all antigens, the proportion of individuals that experienced a decrease in antibody responses range from $73 \%$ to $80 \%$ of individuals in Dielmo and from or $67 \%$ to $76 \%$ of individuals in Ndiop.

Regarding global mean levels of antibody responses (Table 2), the magnitude of decrease in Dielmo was greatest for: $\mathrm{LSA}_{41}$ (- 49\%), followed by $>$ MSP1 $(-35 \%)$, with the smallest decreases against $>$ SE $(-22 \%)$.
In Ndiop, the decay of antibody levels was lower and classification of the magnitude of decrease was largest for: MSP1 $(-21 \%)$ and $>\operatorname{LSA}_{41}(-19 \%)$, and smallest against $>$ SE $(-17 \%)$.

An estimation of the effective loss of IgG levels of young individuals $(<15$ years of age) can be calculated by the difference between the mean antibody levels measured in 2013 and their potential antibody levels with constant transmission i.e. mean antibody responses measured in a comparable $>15$ yo group in 2002 from the entire survey.

In Dielmo, mean levels in $2013(\mathrm{~N}=20$, mean age $=7.7$ in 2002 i.e. 18.8 in 2013) expressed as ODratio and MFI were $3.5,432,386$ (Table 2) compared to expected values of 5.5, 1176, $2395(\mathrm{~N}=44$ individuals, mean age =19.2) for SE, $\mathrm{LSA}_{41}$ and MSP1p19, respectively.

In Ndiop, mean levels in $2013(\mathrm{~N}=28$, mean age $=7.8$ in 2002 i.e. 18.8 in 2013) expressed as ODratio and MFI were $2.5,558,1234$ (Table 2) compared to expected values of $3.8,1913,4132(\mathrm{~N}=44$ individuals, mean age $=18.6$ ) for SE, $\mathrm{LSA}_{41}$ and MSP1p19, respectively.

The potential decreases are $-34 \%,-34 \%$ for SE; $-63 \%,-71 \%$ for $\mathrm{LSA}_{41}$; $-84 \%,-70 \%$ for MSP1p19 for Dielmo and Ndiop, respectively.

\section{Sero-catalytic model and prediction of transmission}

In this analysis, anti-malarial antibody responses were those measured in samples from the 2 cross-sectional studies done before the rainy season in 2002 and 2013 (184 and 196 villagers in Dielmo, and 202 and 216 villagers in Ndiop) [27]. Comparison of levels of prevalence using this set of data are shown in Fig. 3. 

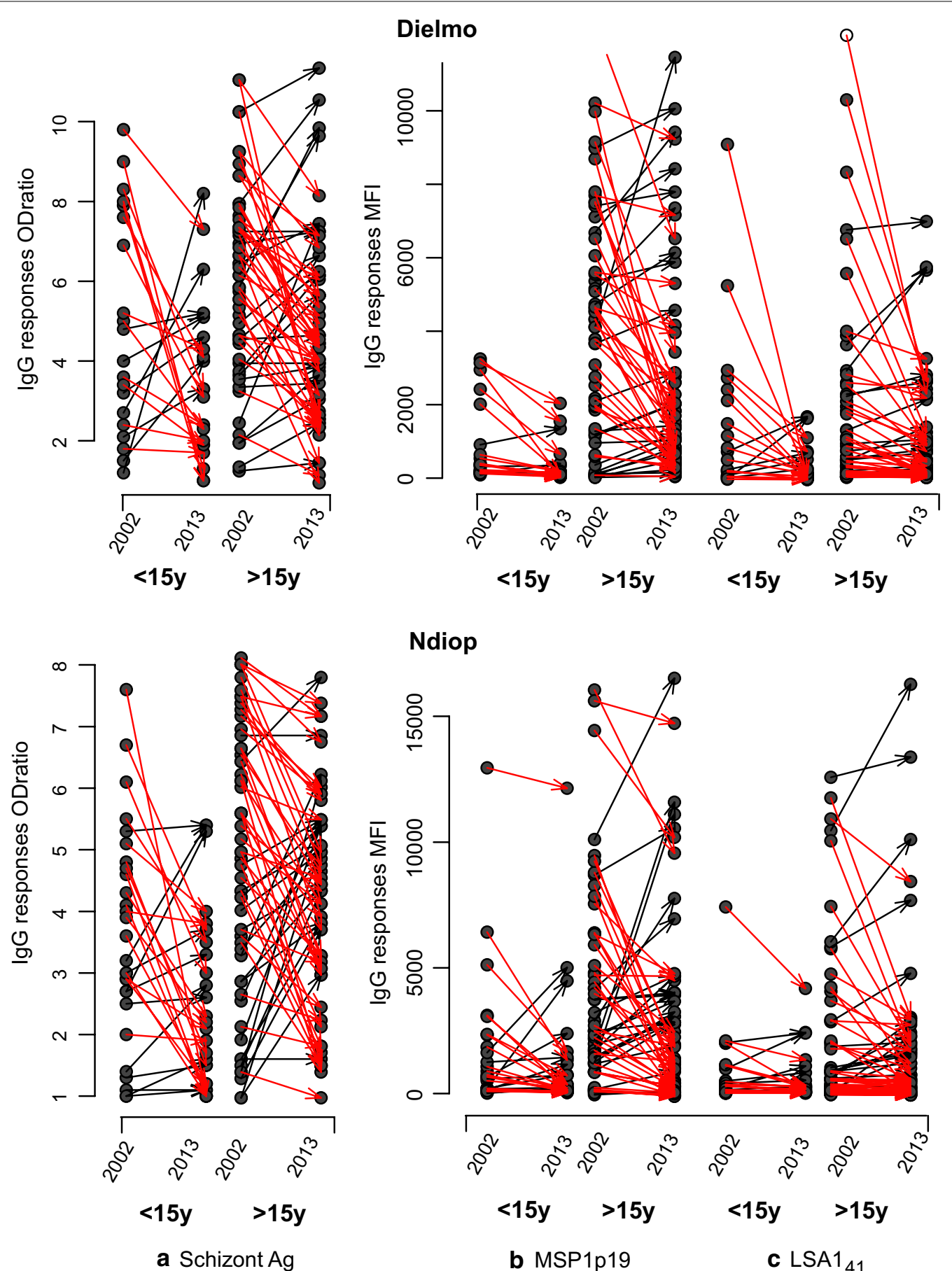

Fig. 2 Individual variations of antibody responses to SE (a), MSP1p19 (b) and LSA1 41 (c) in individuals from Dielmo and Ndiop between 2002 and 2013. This figure show detailed individual antibody responses in Dielmo and Ndiop as dot plot in 2002 and 2013. Red arrows link responses from each individual from 2002 to 2013 in groups of younger villagers (<15 years in 2002) and older ones (> 15 years). Arrows linking individual measures that did not decrease between 2002 and 2013 are in black colour. The decrease of antibody levels was significant for all antigens and for all age groups in Dielmo $(p<0.01$, Wilcoxon signed rank test for paired data). In Ndiop, there was a significant decrease for $\lg G$ to SE and MSP1 p19 ( $p<0.05$ ) but not $\mathrm{LSA}_{41}$. The mean decay of Ab levels ranged from 17 to $49 \%$ 


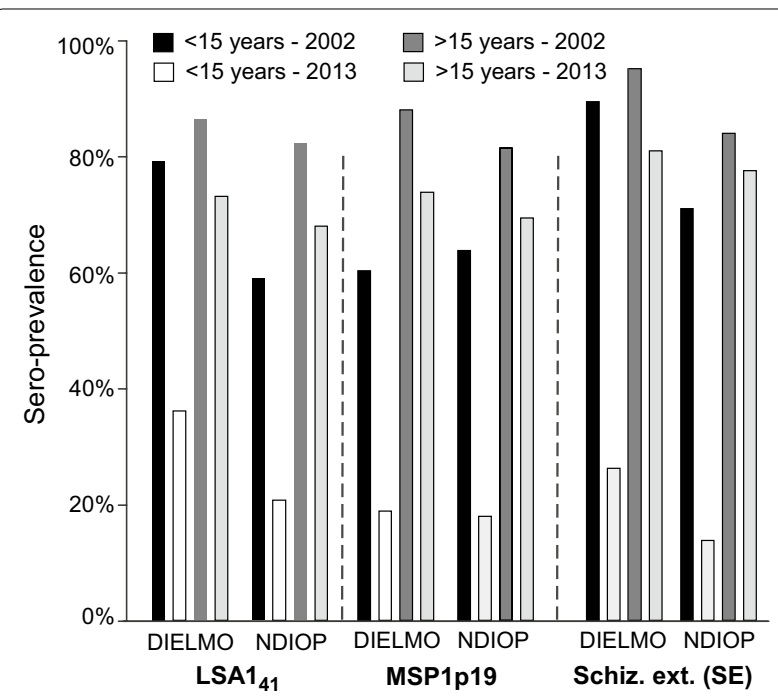

Fig. 3 Prevalence of responders to the antigen tested in $2002 \mathrm{vs}$ 2013. Comparison of prevalence of responders to $\mathrm{LSA}_{41}, \mathrm{MSP} 1 \mathrm{p} 19$ and SE in Dielmo and Ndiop are plotted as vertical bars. The study involved the entire cross-sectional analysis of 184 (Dielmo 2002), 196 (Dielmo 2013), 202 (Ndiop 2002) and 216 villagers (Ndiop 2013) [27]. Plotting of prevalence is shown age-stratified $\leq 15$ years (black and white bars) and $>15$ years (dark and light grey). Classification of the magnitude of decrease (Dielmo, Ndiop, respectively) was: $\mathrm{LSA1}_{41}(54 \%, 65 \%)<\mathrm{MSP} 1 \mathrm{p} 19(69 \%, 72 \%)<\mathrm{SE}(71 \%, 80 \%)$ for the younger age group. In older individuals, decrease of prevalence was around 15\% (8-17\%) without clear difference between prevalence of responses to the 3 antigens

The age-dependent variation in sero-prevalence levels can be formally analysed using sero-catalytic models [7]. Figure 4 shows of such an analysis using the data for LSA $1_{41}$, MSP1p19, and SE with the Dielmo-Ndiop crosssectional surveys from 2002 and 2013.

In both Dielmo and Ndiop, there was a significant substantial reduction in sero-prevalence from 2002 to 2013. The difference in sero-prevalence is most notable in children younger than 10 years of age, where very low levels are observed in the 2013 cross-section. It has been documented that malaria transmission has been historically higher in Dielmo than Ndiop. However, for antibody responses to most antigens, there were not substantial differences in sero-positivity (Additional file 2: Fig. S1), or in model-estimated sero-conversion rate between Dielmo and Ndiop (Additional file 1: Table S1, Additional file 2: Fig. S1). The important exceptions are liverstage antigen 3 (LSA-3), erythrocyte membrane protein 1 (PfEMP1/PF13) and schizont extract (SE) where seropositivity (and hence the model-estimated sero-conversion rates) were higher in Dielmo than in Ndiop.

\section{Discussion}

In the study of patients from Côte d'Ivoire, parasite invasion strongly stimulates immune responses and antibody responses may be higher in more exposed persons who, therefore, have a higher degree of acquired immunity [37]. Cross-sectional analysis of randomly recruited symptomatic and well-documented cases from sentinel sites, showed that a multi-target measure of antibody responses could constitute a surrogate of actual immune status [25]. Here, IgG responses to SE, MSP1 and $\mathrm{LSA}_{41}$ in sentinel sites were compared with results from asymptomatic recruitment (AR) in Abobo and severe malaria cases (SM) from the CHU of Abidjan (Table 1).

When using this limited set of potential markers for immunity in patients with clinical malaria, significant unexpected differences in antibody responses were found depending on geographical setting and clinical status. While patients from the Sentinel National Network health centre showed a clearly augmented antibody response to MSP1p19 and LSA $1_{41}$ in the Korhogo cohort [25], asymptomatic carriers and young individuals with SM had comparable high levels of antibodies to MSP1p19 but variable levels to $\mathrm{LSA}_{41}$. SE antigen was moderately associated with setting except for asymptomatic carriers with lower levels of IgG. Thus, antibody responses measured during a mild clinical episode could represent a surrogate of effective immunity which may depend on the duration and intensity of parasitaemia before treatment. For a given health centre, patients can be considered to have comparable susceptibility to clinical infections requiring hospitalization and treatment, independent of the setting and the individual's previous history of infection. It must be noted recruitment in urban central hospitals are based on symptomatic cases presenting at the facilities, and cross-sectional representative surveys are more likely to capture asymptomatic cases with different immune profiles. Thus, cross sectional surveillance of symptomatic immune responses to selected malarial antigens in local health centres could be a convenient way of assessing immunity at the population level in different areas with high levels of transmission. Such immunity is related to the degree of adherence to control measures, and analysis of antibody responses to validated antigens could be an indirect way of tracking compliance to measures such as LLINs which is the largest contributor to sustained protection [2]. Importantly, immunity affects treatment outcome with a significant impact when using the current first-line artemisinin combined treatments [38]. Monitoring of parasite clearance times in symptomatic malaria, which is routinely done in Côte d'Ivoire, is 

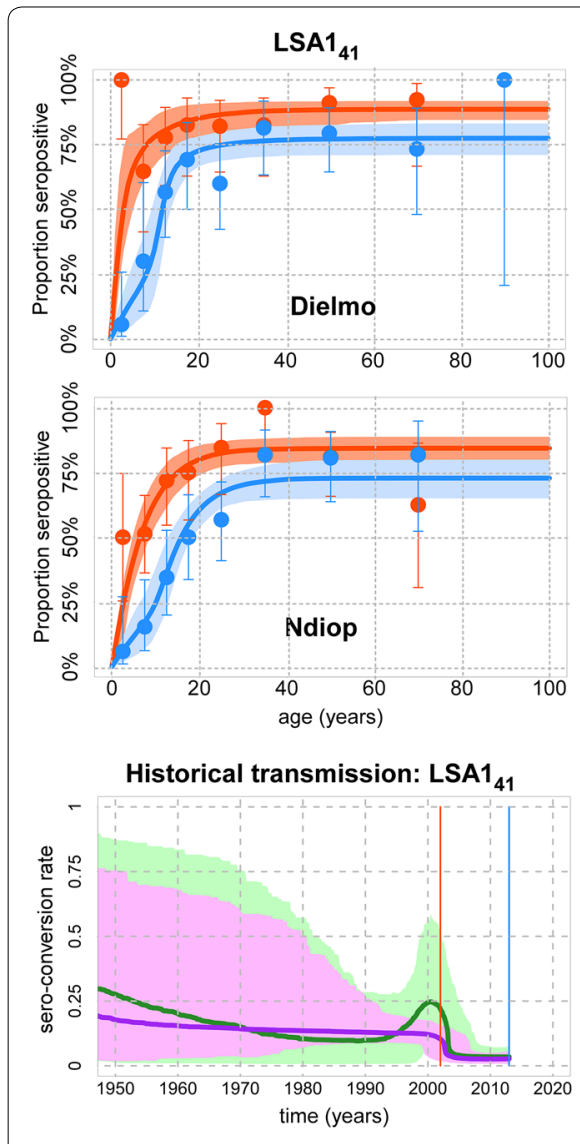

2002 cross-section
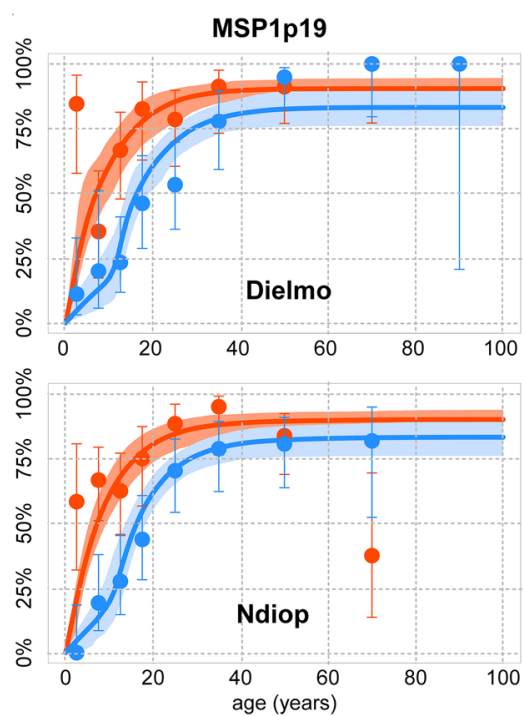

Historical transmission: MSP1p19

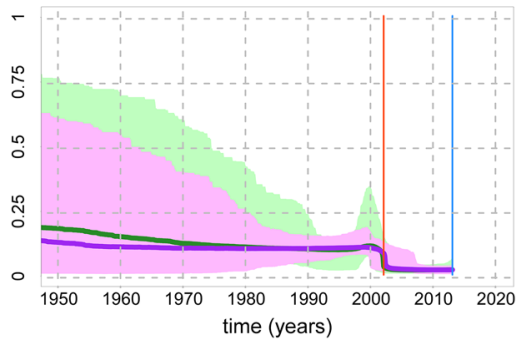

- Dielmo
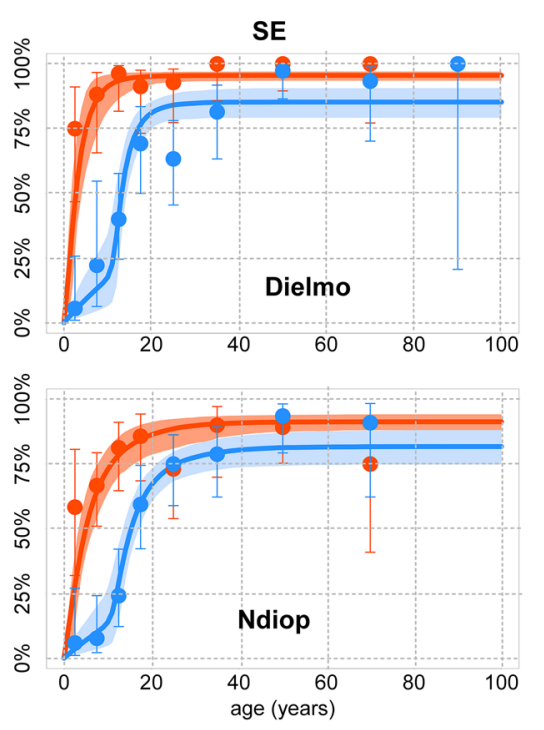

Historical transmission: SE

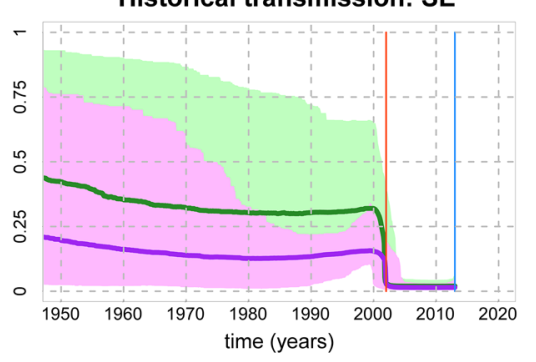

- Ndiop

Fig. 4 Sero-catalytic models fitted to data from cross sectional studies done in 2002 and 2013. Age-stratified sero-prevalence of anti-malarial antibodies to $\mathrm{LSA}_{41}, \mathrm{MSP} 1$ p1 9 and SE in Dielmo (top row) and Ndiop (middle row). Data are plotted as points with vertical bars representing $95 \%$ confidence intervals. The fitted lines represent the posterior median predictions of the sero-catalytic model, and the shaded region denotes the 95\% credible interval. The bottom row shows the estimated historical trends in transmission as measured by changes in sero-conversion rate. The times of the cross-sections are indicated with vertical lines in 2002 (red) and 2013 (blue). Shaded regions denote the 95\% credible interval

a complementary approach for assessing immunity. The establishment of such annual prospective multi-centre cross-sectional studies should help document the effectiveness of large-scale integrated long-lasting control interventions at the community level.

In the cross-sectional studies from the villages of Dielmo and Ndiop done in 2002 and 2013, 75 and 86 villagers respectively, aged from 3.4 to 80 years were evaluated both in 2002 and 2013. During that time, cumulative EIR dropped dramatically from 215 and 171 to 7.5 and 2.5 infective bites/person/year in Dielmo and Ndiop, respectively. The cumulative number of infective bites/person/ year from 2002 to 2008 were 1604, 218 and from 2009 to July 2013 229, 27 in Dielmo and Ndiop, respectively, i.e. a decrease of around $86 \%$ in transmission intensity [6]. Results showed larger decreases of IgG to all antigens in the younger group of 2002 than the older groups. Importantly, the reduced immunity with regard to antibody levels in the younger age group between 2002 and 2013 are substantially higher than reported in Table 2 when estimated by the difference between their actual antibody level measured in 2013 and the expected responses with no reduction in transmission. The decay of IgG levels was $34 \%, 63 \%, 84 \%$ in Dielmo and 34\%, 71\%, $70 \%$ in Ndiop for $\mathrm{SE}, \mathrm{LSA}_{41}$ and MSP1p19, respectively.

These observations suggest that the waning of antibody responses measured using these antigens as markers was much more dramatic for younger individuals in the process of acquiring immunity despite 8.9 to 20.3 years of cumulative unchanged exposure from 2002 to 2008 and 5.5 years decay from 2008 to 2013 . A confounding factor may be the high levels of parasite prevalence in children under 5 years in the 2002 cross-section from Dielmo [27]. Another limitation was also that simultaneous sampling before the peak of transmission was considered as a relatively 'steady' state for IG responses before the 
high fluctuations due to transmission in the rainy season that was high in 2002 and much lower in 2013. Individuals with circulating blood-stage parasites are likely to have also higher levels of circulating IgG antibodies. The individual decrease of antibody responses appears to be a complex pattern depending on previous cumulative and possible recurrent exposure after 2008 when transmission had almost disappeared. The observed increase of specific IgG in some individuals did not correlate between the antigens tested. There were 39/75 villagers in Dielmo and 47/86 in Ndiop with black arrows in Fig. 2, meaning that over 50\% of individuals (mostly adults) showed higher responses in 2013 than in 2002 although less than $7 \%$ had simultaneous increases of IgG to all three antigens. It is clear that the changing epidemiology of infection and disease cannot be easily explained by increased vector control alone. Transient peaks of IgG can result from rebounds in transmission linked to different events such as reduced efficiency of LLINs requiring replacement at least every 3 years [5, 39], climate change [40] or unexplained/undetected asymptomatic submicroscopic carriage [41].

Once transmission has declined, sero-surveillance helps to identify populations where transmission still occurs, allowing targeted interventions. Thus, MSP1p19 appears to be a relevant antigen for detecting IgG signaling parasite presence. It is applicable both to asymptomatic and symptomatic malaria, for example allowing categorization of transmission into two major groups among the complex epidemiological strata in Cameroon [42].

When anti-malarial antibody responses are measured in samples from cross-sectional studies, changes in seroprevalence levels with age can be used to make inferences on changing transmission [27]. For example, if high levels of sero-prevalence are observed in individuals older than 5 years of age, and if very low levels in children less than 5 years, this would suggest that there was a sharp reduction in transmission 5 years previously. The changes in sero-prevalence with age have been used to identify changing trends in transmission. For the three antigens, it is clear that the reduction in transmission occurred after the 2002 survey. Over the period of time where data has been collected, $P$. falciparum prevalence and incidence have been substantially higher in Dielmo compared to Ndiop. Anti-SE sero-prevalence levels reflect this pattern with higher levels of sero-prevalence in Dielmo than in Ndiop. However, levels of anti-LSA $1_{41}$ and anti-MSP1p19 sero-prevalence are comparable between Ndiop and Dielmo. There is an important need to identify antigens or combinations of antigens that best reflect known transmission trends.

\section{Conclusion}

The goal is to identify a limited number of relevant antigens to develop standard screening methods for monitoring the antibody-related immune levels of populations residing in regions where the incidence of clinical malaria remains high or proceeding towards pre-elimination. As a simple ELISA-based technology for measuring antibody responses to multiple antigens, the multiplex approach offers the potential to generate important epidemiologic surveillance data accessible to malaria control programmes.

\section{Supplementary information}

Supplementary information accompanies this paper at https://doi. org/10.1186/s12936-020-03186-9.

Additional file 1: Table S1. Estimates from sero-catalytic models. Statistical inference was implemented in a Bayesian framework with uniform priors. Parameters are presented as median and 95\% credible intervals of the estimated posterior distributions.

Additional file 2: Fig. S1. Sero-catalytic models fitted to data from antibody responses to eight antigens plus schizont extract measured in cross sectional studies done in 2002 and 2013.Age-stratified sero-prevalence of anti-malarial antibodies in Dielmo and Ndiop. Data are plotted as points with vertical bars representing $95 \%$ confidence intervals. The fitted lines represent the posterior median predictions of the sero-catalytic model, and the shaded region denotes the $95 \%$ credible interval. The third and sixth row shows the estimated historical trends in transmission as measured by changes in sero-conversion rate. Shaded regions denote the $95 \%$ credible interval.

\section{Abbreviations}

MFI: Mean fluorescence intensity; ELISA: Enzyme linked immunosorbent assay; MSP1 19: P. falciparum merozoite surface protein 1-19kd fragment; LSA1: Liver stage antigen 1; SE: P. falciparum schizont extract; CHU: University Hospital Centre; MBA: Multiplex bead-based assay; SC: Sero-conversion rate; SRR: Sero-reversion rate; AR: Asymptomatic recruitment; SM: Severe malaria cases; LLINs: Long lasting impregnated bed nets; ACT: Artemisinin-based combination therapy; EIR: Entomological inoculation rate; IgG: Immunoglobulin G.

\section{Acknowledgements}

We are particularly grateful to Dr Shirley Longacre (Vaximax, Paris) who generously provided the baculovirus Plasmodium falciparum MSP1p19 antigen. We thank gratefully Drs Odile Mercereau-Puijalon, Aissatou Touré and Pr Alioune Dieye for constant support, Joseph Faye for Dielmo/Ndiop database management and Cheikh Loucoubar for bioinformatics support. We are indebted to Dr Jean-François Trape and Cheikh Sokhna (IRD, Dakar) for their institutional support in the follow-up of Dielmo and Ndiop villages. We thank gratefully all villagers from Dielmo and Ndiop for their generous and committed long-term participation to the study. We thank all patients from Cote d'Ivoire for their participation to the survey.

\section{Authors' contributions}

RP, AO and MLV designed the study. FDS, DK, AO, MLV supervised the collection of field samples and clinical data. MLV, DK, RP, conducted the laboratory experiments and statistical analyses, with help and input from MN and BM and FDS. Advanced statistics and mathematical models were done by MW, analysis of data were done by RP, MLV, BM. MW, MLV and RP drafted the paper, with input and comments from all other authors. Figures and tables were prepared by RP, MW and MLV with input from all authors. All authors read and approved the final manuscript. 


\section{Funding}

The work was supported by grants from the Institut Pasteur Foundation, the prix Jacques Piraud of the Fondation pour la Recherche Médicale and grants from Institut Pasteur ACIP 25_2012 and from the Rotary International associated with the Rotary Paris Alliance and Rotary Dakar Almadies.

\section{Availability of data and materials}

Not applicable.

\section{Ethics approval and consent to participate}

In Sénégal, the follow-up in the endemic villages of Dielmo and Ndiop is a long-term longitudinal survey to study acquisition and maintenance of natural immunity. The Dielmo/Ndiop project has been carried out over 25 years and presently ongoing. The project protocol and objectives were carefully explained to the assembled villagers, and informed written consent was obtained from all participants or their parents or guardians. Consent is individually renewed from all subjects; anyone can withdraw from the study and the follow-up procedure at any time. The protocol was approved by the Senegalese National Health Research Ethics Committee. An agreement between the Fondation Institut Pasteur de Dakar, Institut de Recherche pour le Développement (IRD) and the Ministère de la Santé et de la Prévention of Senegal defines all research activities.In Côte d'Ivoire, the protocol of surveillance was approved by the National Committee of the Ministry of Health taking place in the national procedure of malaria surveillance in health centre of the country. Individual informed written consent was obtained from participants/parents/ guardians. In case of an illiterate patient, his/her thumb impression and signature of an independent witness were obtained. The study was conducted in accordance with the local laws and regulations, International Conference on Harmonization-Good Clinical Practice (ICH-GCP). The protocol was reviewed and approved by the Comité National d'Ethique et de Recherche de Côte d'Ivoire (N56/MSLS/CNER-dkn). All clinical cases were treated and followedup according to the standard national procedure.

\section{Consent for publication}

Not applicable.

\section{Competing interests}

The authors declare no competing interests that exist.

\section{Author details}

${ }^{1}$ Unité d'Immunologie, Institut Pasteur de Dakar, Dakar, Senegal. ${ }^{2}$ Unité de Paludologie, Institut Pasteur de Côte d'Ivoire, Abidjan, Côte d'Ivoire. ${ }^{3}$ Malaria Unit, Parasites and hosts, Institut Pasteur, Paris, France. ${ }^{4}$ Unité d'Epidémiologie, Institut Pasteur de Dakar, Dakar, Senegal. ${ }^{5}$ Unité d'Immunogénétique, Institut Pasteur de Dakar, Dakar, Senegal. ${ }^{6}$ Service d'Immunologie FMPO, Université Cheikh Anta Diop de Dakar, Dakar, Senegal. ${ }^{7}$ Present Address: Centre Pasteur du Cameroun, Annexe de Garoua, Garoua, Cameroun.

Received: 18 June 2019 Accepted: 9 March 2020

Published online: 19 March 2020

\section{References}

1. WHO. World Malaria Report 2016. Geneva: World Health Organization; 2016. http://www.who.int/malaria/publications/world-malaria-repor t-2016/en/ 2016.

2. Bhatt S, Weiss DJ, Cameron E, Bisanzio D, Mappin B, Dalrymple U, et al. The effect of malaria control on Plasmodium falciparum in Africa between 2000 and 2015. Nature. 2015;526:207-11.

3. WHO. World Malaria Report 2008. Geneva: World Health Organization; 2008. http://www.who.int/malaria/publications/atoz/9789241563697/ en/index.html. 2010.

4. Trape JF, Tall A, Diagne N, Ndiath O, Ly AB, Faye J, et al. Malaria morbidity and pyrethroid resistance after the introduction of insecticide-treated bednets and artemisinin-based combination therapies: a longitudinal study. Lancet Infect Dis. 2011;11:925-32.

5. Wotodjo AN, Doucoure S, Gaudart J, Diagne N, Diene Sarr F, Faye N, et al. Malaria in Dielmo, a Senegal village: is its elimination possible after seven years of implementation of long-lasting insecticide-treated nets? PLoS ONE. 2017;12:e0179528.
6. Trape JF, Tall A, Sokhna C, Ly AB, Diagne N, Ndiath O, et al. The rise and fall of malaria in a West African rural community, Dielmo, Senegal, from 1990 to 2012: a 22 year longitudinal study. Lancet Infect Dis. 2014;14:476-88.

7. Corran P, Coleman P, Riley E, Drakeley C. Serology: a robust indicator of malaria transmission intensity? Trends Parasitol. 2007;23:575-82.

8. Crompton PD, Kayala MA, Traore B, Kayentao K, Ongoiba A, Weiss G, et al. A prospective analysis of the Ab response to Plasmodium falciparum before and after a malaria season by protein microarray. Proc Natl Acad Sci USA. 2010;107:6958-63.

9. Drakeley C, Cook J. Potential contribution of sero-epidemiological analysis for monitoring malaria control and elimination: historical and current perspectives. Adv Parasitol. 2009;69:299-352.

10. Elliott SR, Fowkes FJ, Richards JS, Reiling L, Drew DR, Beeson JG. Research priorities for the development and implementation of serological tools for malaria surveillance. F1000 Prime Rep. 2014;6:100.

11. Osier FH, Fegan G, Polley SD, Murungi L, Verra F, Tetteh KK, et al. Breadth and magnitude of antibody responses to multiple Plasmodium falciparum merozoite antigens are associated with protection from clinical malaria. Infect Immun. 2008;76:2240-8.

12. Richards JS, Arumugam TU, Reiling L, Healer J, Hodder AN, Fowkes FJ, et al. Identification and prioritization of merozoite antigens as targets of protective human immunity to Plasmodium falciparum malaria for vaccine and biomarker development. J Immunol. 2013;191:795-809.

13. Oeuvray C, Theisen M, Rogier C, Trape JF, Jepsen S, Druilhe P. Cytophilic immunoglobulin responses to Plasmodium falciparum glutamate-rich protein are correlated with protection against clinical malaria in Dielmo. Senegal. Infect Immun. 2000;68:2617-20.

14. Perraut R, Joos C, Sokhna C, Polson HE, Trape JF, Tall A, et al. Association of antibody responses to the conserved Plasmodium falciparum Merozoite Surface Protein 5 with protection against clinical malaria. PLOS ONE. 2014:9:e101737.

15. Perraut R, Marrama L, Diouf B, Sokhna C, Tall A, Nabeth P, et al. Antibodies to the conserved $C$-terminal domain of the Plasmodium falciparum merozoite surface protein 1 and to the merozoite extract and their relationship with in vitro inhibitory antibodies and protection against clinical malaria in a Senegalese village. J Infect Dis. 2005;191:264-71.

16. Perraut R, Varela ML, Joos C, Diouf B, Sokhna C, Mbengue B, et al. Association of antibodies to Plasmodium falciparum merozoite surface protein-4 with protection against clinical malaria. Vaccine. 2017;35:6720-6.

17. Roussilhon C, Oeuvray C, Muller-Graf C, Tall A, Rogier C, Trape JF, et al. Long-term clinical protection from falciparum malaria is strongly associated with lgG3 antibodies to merozoite surface protein 3. PLoS Med. 2007:4:e320.

18. Toure-Balde A, Perlaza BL, Sauzet JP, Ndiaye M, Aribot G, Tall A, et al. Evidence for multiple B- and T-cell epitopes in Plasmodium falciparum liver-stage antigen 3. Infect Immun. 2009;77:1189-96.

19. Dodoo D, Aikins A, Kusi KA, Lamptey H, Remarque E, Milligan P, et al. Cohort study of the association of antibody levels to AMA1, MSP119, MSP3 and GLURP with protection from clinical malaria in Ghanaian children. Malar J. 2008;7:142.

20. Richards JS, Stanisic DI, Fowkes FJ, Tavul L, Dabod E, Thompson JK, et al. Association between naturally acquired antibodies to erythrocyte-binding antigens of Plasmodium falciparum and protection from malaria and high-density parasitaemia. Clin Inf Dis. 2010;51:e50-60.

21. Taylor RR, Allen SJ, Greenwood BM, Riley EM. IgG3 antibodies to Plasmodium falciparum merozoite surface protein 2 (MSP2): increasing prevalence with age and association with clinical immunity to malaria. Am J Trop Med Hyg. 1998;58:406-13.

22. Kinyanjui SM, Bejon P, Osier FH, Bull PC, Marsh K. What you see is not what you get: implications of the brevity of antibody responses to malaria antigens and transmission heterogeneity in longitudinal studies of malaria immunity. Malar J. 2009;8:242.

23. Perraut R, Mercereau-Puijalon O, Diouf B, Tall A, Guillotte M, Le Scanf C, et al. Seasonal fluctuation of antibody levels to Plasmodium falciparum parasitized red blood cell-associated antigens in two Senegalese villages with different transmission conditions. Am J Trop Med Hyg. 2000;62:746-51.

24. Helb DA, Tetteh KK, Felgner PL, Skinner J, Hubbard A, Arinaitwe E, et al. Novel serologic biomarkers provide accurate estimates of recent Plasmodium falciparum exposure for individuals and communities. Proc Natl Acad Sci USA. 2015:112:E4438-47. 
25. Koffi D, Toure AO, Varela ML, Vigan-Womas I, Beourou S, Brou S, et al. Analysis of antibody profiles in symptomatic malaria in three sentinel sites of Ivory Coast by using multiplex, fluorescent, magnetic, bead-based serological assay (MAGPIX). Malar J. 2015;14:509.

26. Koffi D, Varela ML, Loucoubar C, Beourou S, Vigan-Womas I, Toure A, et al. Longitudinal analysis of antibody responses in symptomatic malaria cases do not mirror parasite transmission in peri-urban area of Côte d'Ivoire between 2010 and 2013. PLoS ONE. 2017;12:e0172899.

27. Perraut R, Varela ML, Loucoubar C, Niass O, Sidibe A, Tall A, et al. Serological signatures of declining exposure following intensification of integrated malaria control in two rural Senegalese communities. PLoS ONE. 2017;12:e0179146.

28. Perraut R, Guillotte M, Drame I, Diouf B, Molez JF, Tall A, et al. Evaluation of anti-Plasmodium falciparum antibodies in Senegalese adults using different types of crude extracts from various strains of parasite. Microbes Infect. 2002:4:31-5.

29. Betsi AN. La riziculture et le paludisme dans la région forestière ouest de la Côte d'Ivoire. Cah Agricultures. 2003:12:341-6.

30. Trape JF, Rogier C. Combating malaria morbidity and mortality by reducing transmission. Parasitol Today. 1996:12:236-40.

31. Diop F, Diop G, Niang M, Diouf B, Ndiaye D, Richard V, et al. The value of local malaria strains for serological studies: local strains versus Palo Alto reference strain. Malar J. 2015;14:229.

32. Perraut R, Marrama L, Diouf B, Fontenille D, Tall A, Sokhna C, et al. Distinct surrogate markers for protection against Plasmodium falciparum infection and clinical malaria identified in a Senegalese community after radical drug cure. J Infect Dis. 2003;188:1940-50.

33. Ambrosino E, Dumoulin C, Orlandi-Pradines E, Remoue F, Toure-Balde A, Tall A, et al. A multiplex assay for the simultaneous detection of antibodies against 15 Plasmodium falciparum and Anopheles gambiae saliva antigens. Malar J. 2010;9:317.

34. Kerkhof K, Sluydts V, Willen L, Kim S, Canier L, Heng S, et al. Serological markers to measure recent changes in malaria at population level in Cambodia. Malar J. 2016;15:529.

35. Perraut R, Richard V, Varela ML, Trape JF, Guillotte M, Tall A, et al. Comparative analysis of IgG responses to Plasmodium falciparum MSP1p19 and PF13-DBL1 alpha1 using ELISA and a magnetic bead-based duplex assay
(MAGPIX(R)-Luminex) in a Senegalese meso-endemic community. Malar J. 2014;13:410.

36. Perraut R, Varela ML, Mbengue B, Guillotte M, Mercereau-Puijalon O, Vigan-Womas I. Standardization of a multiplex magnetic bead-based assay for simultaneous detection of IgG to Plasmodium antigens. J Immunol Tech Infect Dis. 2015;4:1-8.

37. Greenhouse B, Ho B, Hubbard A, Njama-Meya D, Narum DL, Lanar DE et al. Antibodies to Plasmodium falciparum antigens predict a higher risk of malaria but protection from symptoms once parasitemic. J Infect Dis. 2011;204:19-26.

38. O'Flaherty K, Maguire J, Simpson JA, Fowkes FJI. Immunity as a predictor of anti-malarial treatment failure: a systematic review. Malar J. 2017:16:158.

39. Solomon T, Loha E, Deressa W, Balkew M, Gari T, Overgaard HJ, et al. Bed nets used to protect against malaria do not last long in a semi-arid area of Ethiopia: a cohort study. Malar J. 2018;17:239.

40. Ishengoma DS, Mmbando BP, Mandara Cl, Chiduo MG, Francis F, Timiza $W$, et al. Trends of Plasmodium falciparum prevalence in two communities of Muheza district North-eastern Tanzania: correlation between parasite prevalence, malaria interventions and rainfall in the context of re-emergence of malaria after two decades of progressively declining transmission. Malar J. 2018;17:252.

41. Niang M, Thiam LG, Sane R, Diagne N, Talla C, Doucoure S, et al. Substantial asymptomatic submicroscopic Plasmodium carriage during dry season in low transmission areas in Senegal: implications for malaria control and elimination. PLOS ONE. 2017;12:e0182189.

42. Kwenti TE, Moye AL, Wiylanyuy AB, Njunda LA, Nkuo-Akenji T. Variation in the immune responses against Plasmodium falciparum merozoite surface protein-1 and apical membrane antigen-1 in children residing in the different epidemiological strata of malaria in Cameroon. Malar J. 2017:16:453.

\section{Publisher's Note}

Springer Nature remains neutral with regard to jurisdictional claims in published maps and institutional affiliations.
Ready to submit your research? Choose BMC and benefit from:

- fast, convenient online submission

- thorough peer review by experienced researchers in your field

- rapid publication on acceptance

- support for research data, including large and complex data types

- gold Open Access which fosters wider collaboration and increased citations

- maximum visibility for your research: over $100 \mathrm{M}$ website views per year

At BMC, research is always in progress.

Learn more biomedcentral.com/submissions 